\title{
CONSTANT MEAN CURVATURE HYPERSURFACES IN SPHERES
}

\author{
QIN-TAO DENG* \\ Laboratory of Nonlinear Analysis, Huazhong Normal University, Wuhan 430079, P. R. China \\ e-mail: qintaodeng@yahoo.com.cn \\ HUI-LING GU** \\ Department of Mathematics, Sun Yat-Sen University, Guangzhou 510275, P. R. China \\ e-mail:guhuil@mail.sysu.edu.cn \\ and YAN-HUI SU \\ Department of Mathematics, Sun Yat-Sen University, Guangzhou 510275, P. R. China \\ e-mail: r314272@yahoo.com.cn
}

(Received 3 November 2010; revised 24 April 2011; accepted 4 May 2011; first published online 2 August 2011)

\begin{abstract}
In this paper, we first summarise the progress for the famous Chern conjecture, and then we consider $n$-dimensional closed hypersurfaces with constant mean curvature $H$ in the unit sphere $\mathbb{S}^{n+1}$ with $n \leq 8$ and generalise the result of Cheng et al. (Q. M. Cheng, Y. J. He and H. Z. Li, Scalar curvature of hypersurfaces with constant mean curvature in a sphere, Glasg. Math. J. 51(2) (2009), 413-423). In order to be precise, we prove that if $|H| \leq \varepsilon(n)$, then there exists a constant $\delta(n, H)>0$, which depends only on $n$ and $H$, such that if $S_{0} \leq S \leq S_{0}+\delta(n, H)$, then $S=S_{0}$ and $M$ is isometric to the Clifford hypersurface, where $\varepsilon(n)$ is a sufficiently small constant depending on $n$.
\end{abstract}

2010 Mathematics Subject Classification. 53C40, 53C42.

\section{Introduction.}

1.1. Minimal hypersurfaces in $\mathbb{S}^{n+1}$. In this section we will review some important results about the following famous conjecture. For more details, refer to Scherfner and Weiss' [13] excellent survey on this topic.

CHERn CONJECTURE. Let $S$ be the value of the squared norm of the second fundamental forms for n-dimensional closed minimal hypersurfaces in the unit sphere $\mathbb{S}^{n+1}$ with constant scalar curvature, then the set of $S$ should be discrete.

The above conjecture has been studied by many mathematicans after the work of Chern et al. [7]. In 1968, Simons [14] proved the following.

\footnotetext{
* The first author was supported by NSFC (nos. 10901067 and 10801058), the Special Fund for Basic Scientific Research of Central Colleges (no. CCNU10A02015) and Hubei Key Laboratory of Mathematical Sciences.

** The second author was supported by NSFC (no. 10901165) and the Fundamental Research Funds for the Central Universities (no. 101gpy25) and China Post-doctoral Science Foundation (no. 201003382).
} 
THEOREM 1.1 ([14]). Let $M^{n}$ be an n-dimensional closed minimal hypersurface in a unit sphere $\mathbb{S}^{n+1}$, then

$$
\int_{M^{n}} S(S-n) \geq 0
$$

where $S$ denotes the squared norm of the second fundamental form of $M^{n}$.

From Theorem 1.1, we immediately conclude that if $0 \leq S \leq n$, then either $S \equiv 0$ or $S \equiv n$. The latter case was independently characterised by Chern et al. [7] and Lawson [9].

THEOREM $1.2(7,9)$. The Clifford tori are the only closed minimal hypersurfaces in $\mathbb{S}^{n+1}$ with $S=n$.

According to the example given by Cartan [3], the next value of $S$ may be $2 n$. Peng and Terng [11] considered the next value of $S$ and proved the following theorem.

THEOREM 1.3 ([11]). Let $M^{n}(n \geq 3)$ be a closed minimal hypersurface in $\mathbb{S}^{n+1}$ with $S=$ constant. If $S>n$, then

$$
S>n+\frac{1}{12 n}
$$

Moreover, for the case of $n=3$, Peng-Terng obtained the following sharp result.

THEOREM 1.4 ([11]). Let $M^{3}$ be a closed minimal hypersurface in $\mathbb{S}^{4}$ with $S=$ constant. If $S>3$, then $S \geq 6$. Moreover, $S=6$ is assumed in the examples of Cartan [3] and Hsiang [8].

Chang [5] proved the following classification theorem for $n=3$. Thus, the Chern conjecture [7] is right for $n=3$.

THEOREM 1.5 (Chang's Classification Theorem [5]). A closed minimally immersed hypersurface with constant scalar curvature in $\mathbb{S}^{4}$ is either an equatorial 3-sphere, a product of sphere, or a Cartan's minimal hypersurface.

In particular, $S$ can only be $0,3,6$.

For the closed hypersurface $M^{3}$ of $\mathbb{S}^{4}$ with constant mean curvature and scalar curvature, Almeida-Brito [1] and Chang [4] proved that $M^{3}$ is isoparametric. There is another recent result by Almeida et al. [2], which is as follows:

THEOREM 1.6 ([2]). Let $H, K$ and $R$ be the mean curvature, the Gauss-Kronecker curvature and scalar curvature of $M^{3}$. If two out of these three functions are constant, then either (1) $M^{3}$ is an isoparametric hypersurface of $\mathbb{S}^{4}$, or (2) $H=K \equiv 0$.

However, if $M^{3}$ is not closed, then we have the following conjecture, which is still open. 
BRYANT CONJECTURE. A piece of a minimally immersed hypersurface of constant scalar curvature in $\mathbb{S}^{4}$ is isoparametric.

For the case $n=4$, Lusala et al. [10] proved the following:

THEOREM 1.7 ([10]). A closed minimal Willmore hypersurface $M^{4}$ of $\mathbb{S}^{5}$ with nonnegative constant scalar curvature must be isoparametric.

For general $n$, Cheng and Yang improved Theorem 1.3 as follows (please see [17-19] for details).

THEOREM 1.8 ([17-19]). Let $M^{n}(n>3)$ be a closed minimal hypersurface in $\mathbb{S}^{n+1}$ with $S=$ constant. If $S>n$, then

$$
S>n+\frac{n}{3}
$$

By using the method of Cheng-Yang and by carefully estimation, Suh-Yang [15] improved Cheng-Yang's result as follows.

THEOREM $1.9([\mathbf{1 5}])$. Let $M^{n}(n>3)$ be a closed minimal hypersurface in $\mathbb{S}^{n+1}$ with $S=$ constant. If $S>n$, then

$$
S>n+\frac{3 n}{7}
$$

Until now, we still have the following open problem.

Open problem. Let $M^{n}(n>3)$ be a closed minimal hypersurface in $\mathbb{S}^{n+1}$ with $S=$ constant. If $S>n$, then $S \geq 2 n$ ?

If we do not add the condition that $M^{n}$ has constant scalar curvature, then the following is obtained.

THEOREM 1.10 ([12]). Let $M$ be a closed minimally immersed hypersuface in $\mathbb{S}^{n+1}$, $n \leq 5$ and $S$ the square of the length of the second fundamental form of $M$. Then there exists $\delta(n)>0$ such that if $n \leq S(x)<n+\delta(n)$, then $S(x) \equiv n$, hence $M$ is the Clifford torus.

In [16], Wei and $\mathrm{Xu}$ improved the above theorem from $n \leq 5$ to $n \leq 7$, and then Zhang [20] extented it to $n \leq 8$.

1.2. Constant mean curvature hypersurfaces in $\mathbb{S}^{n+1}$. For constant mean curvature hypersurfaces in $\mathbb{S}^{n+1}$, we will prove the following theorem, which is corresponding to Theorem 1.10.

THEOREM 1.11. Let $M^{n}$ be an $n$-dimensional $(n \leq 8)$ closed hypersurface with constant mean curvature $H$ in $\mathbb{S}^{n+1}(1)$ and $S$ be the length of the second fundamental form of $M^{n}$. Then there exist positive constants $\varepsilon(n)$ depending only on $n$ and $\delta(n, H)$ depending only on $n$ and $H$ such that if

$$
|H| \leq \varepsilon(n) \quad \text { and } \quad S_{0} \leq S \leq S_{0}+\delta(n, H),
$$


where

$$
S_{0}=n+\frac{n^{3}}{2(n-1)} H^{2}+\frac{n(n-2)}{2(n-1)} \sqrt{n^{2} H^{4}+4(n-1) H^{2}},
$$

then $S \equiv S_{0}$ and $M^{n}$ is isometric to the Clifford hypersurface. To be precise, $M^{n}$ is isometric to the Clifford torus $\mathbb{S}^{k}\left(\sqrt{\frac{k}{n}}\right) \times \mathbb{S}^{n-k}\left(\sqrt{\frac{n-k}{n}}\right)$ if $H=0 ; M^{n}$ is isometric to the Clifford hypersurface $\mathbb{S}^{1}\left(\frac{1}{\sqrt{1+\lambda^{2}}}\right) \times \mathbb{S}^{n-1}\left(\frac{\lambda}{\sqrt{1+\lambda^{2}}}\right)$ if $H \neq 0$.

REMARK 1.12. In [6], Cheng et al. proved the above theorem for $n \leq 7$.

2. Basic formulas for closed hypersurfaces with constant mean curvature $H$ in $\mathbb{S}^{n+1}(1)$. In this section we recall basic formulas for closed hypersurfaces with constant mean curvature $H$ in $\mathbb{S}^{n+1}(1)$, which can be found in [6].

Let $M^{n}$ be an $n$-dimensional closed hypersurface with constant mean curvature $H$ in $\mathbb{S}^{n+1}(1)$. Then we choose a local orthonormal frame field $\left\{e_{1}, e_{2}, \ldots, e_{n}\right.$, $\left.e_{n+1}\right\}$ such that $\left\{e_{1}, e_{2}, \ldots, e_{n}\right\}$ is tangent to $M^{n}$ when restricted to $M^{n}$. Let $h_{i j}$ and $H$ denote the second fundamental form and mean curvature, respectively. Denote

$$
S=\sum_{i, j} h_{i j}^{2}, \quad H=\frac{1}{n} \sum_{i} h_{i i}, \quad f_{3}=\sum_{i, j, k} h_{i j} h_{j k} h_{k i}, \quad f_{4}=\sum_{i, j, k, l} h_{i j} h_{j k} h_{k l} h_{l i} .
$$

Denote by $h_{i j k}$ and $h_{i j k l}$ components of the first and second covariant derivatives of the second fundamental form, respectively. For an arbitrary fixed point $p \in M$, we take orthonormal frames such that $h_{i j}=\lambda_{i} \delta_{i j}$ at $p$, for all $i, j$. Then at this point $p$, we have

$$
S=\sum_{i=1}^{n} \lambda_{i}^{2}, \quad H=\frac{1}{n} \sum_{i=1}^{n} \lambda_{i}, \quad f_{3}=\sum_{i=1}^{n} \lambda_{i}^{3}, \quad f_{4}=\sum_{i=1}^{n} \lambda_{i}^{4} .
$$

We define $A, B$ by

$$
A=\sum_{i, j, k} h_{i j k}^{2} \lambda_{i}^{2} \text { and } B=\sum_{i, j, k} h_{i j k}^{2} \lambda_{i} \lambda_{j}
$$

Then by some computation, we have the following formulas:

$$
\begin{aligned}
\frac{1}{2} \Delta S= & S(n-S)-n^{2} H^{2}+n H f_{3}+\sum_{i, j, k} h_{i j k}^{2}, \\
\frac{1}{2} \Delta \sum_{i, j, k} h_{i j k}^{2}= & (2 n+3-S) \sum_{i, j, k} h_{i j k}^{2}-3(A-2 B) \\
& -\frac{3}{2}|\nabla S|^{2}+3 n H \sum_{i, j, k} h_{i j k}^{2} \lambda_{i}+\sum_{i, j, k, l} h_{i j k l}^{2}, \\
\triangle f_{3}= & 3(n-S) f_{3}+3 n H f_{4}-3 n H S+6 \sum_{i, j, k} h_{i j k}^{2} \lambda_{i}, \\
\int_{M} A-2 B= & \int_{M} S f_{4}-f_{3}^{2}-S^{2}+n H f_{3}-\frac{|\nabla S|^{2}}{4} .
\end{aligned}
$$




\section{Proof of the theorem.}

Lemma 3.1. Let $\lambda_{1}, \lambda_{2}, \ldots, \lambda_{n}$ be real numbers. Denote

$$
\begin{aligned}
& \sum_{i=1}^{n} \lambda_{i}=n H, \quad \sum_{i=1}^{n} \lambda_{i}^{2}=S, \\
& \sum_{i=1}^{n} \lambda_{i}^{3}=f_{3}, \quad \sum_{i=1}^{n} \lambda_{i}^{4}=f_{4} .
\end{aligned}
$$

Let $a_{i j}(1 \leq i \leq n, 1 \leq j \leq n)$ be real numbers satisfying

$$
\sum_{j=1}^{n} a_{i j}=\frac{1}{2}\left[(n-S) \lambda_{i}+n H \lambda_{i}^{2}-n H\right], \quad a_{i j}=a_{j i} .
$$

Then

$$
\sum_{i=1}^{n} a_{i i}^{2}+3 \sum_{i \neq j} a_{i j}^{2} \geq \frac{3}{2(n+4)}\left[(n-S)^{2} S+n^{2} H^{2} f_{4}-n^{3} H^{2}+2(n-S) n H f_{3}\right] .
$$

Proof. Consider the function

$$
\begin{aligned}
f\left(a_{i j}, \alpha_{i}, \beta_{i j}\right)= & \sum_{i=1}^{n} a_{i i}^{2}+3 \sum_{i \neq j} a_{i j}^{2}+\sum_{i \neq j} \beta_{i j}\left(a_{i j}-a_{j i}\right) \\
& +\sum_{i=1}^{n} \alpha_{i}\left(\sum_{j=1}^{n} a_{i j}-\frac{1}{2}\left[(n-S) \lambda_{i}-n H \lambda_{i}^{2}+n H\right]\right) .
\end{aligned}
$$

Then by direct computation, we can obtain

$$
\begin{aligned}
\frac{\partial f}{\partial a_{i i}} & =2 a_{i i}+\alpha_{i} \quad(1 \leq i \leq n), \\
\frac{\partial f}{\partial a_{i j}} & =6 a_{i j}+\alpha_{i}+\beta_{i j}-\beta_{j i} \quad(i \neq j) .
\end{aligned}
$$

According to the method of Lagrange multipliers, we solve the equations

$$
\frac{\partial f}{\partial a_{i i}}=0, \quad \frac{\partial f}{\partial a_{i j}}=0,
$$

and obtain

$$
\begin{array}{r}
\alpha_{i}=-\frac{6}{n+4}\left[(n-S) \lambda_{i}+n H \lambda_{i}^{2}-n H\right], \\
a_{i i}=-\frac{\alpha_{i}}{2}, \quad a_{i j}=-\frac{\alpha_{i}+\alpha_{j}}{12}(i \neq j) .
\end{array}
$$

Put the expressions of $a_{i i}$ and $a_{i j}$ into $\sum_{i=1}^{n} a_{i i}^{2}+3 \sum_{i \neq j} a_{i j}^{2}$, and we get the minimum. Hence, we finished the proof of the lemma. 
LEMMA 3.2. Let $M^{n}$ be a closed hypersurface with constant mean curvature $H$ in $\mathbb{S}^{n+1}$. Then

$$
\begin{aligned}
\sum_{i, j, k, l} h_{i j k l}^{2} \geq & \frac{3}{2}\left(S f_{4}-f_{3}^{2}-S^{2}-S(S-n)-n^{2} H^{2}+2 n H f_{3}\right) \\
& +\frac{3}{2(n+4)}\left[(n-S)^{2} S+n^{2} H^{2} f_{4}-n^{3} H^{2}+2(n-S) n H f_{3}\right]
\end{aligned}
$$

Proof. Since

$$
\begin{aligned}
\sum_{i, j, k, l}\left(h_{i j k l}-h_{i j l k}\right)^{2} & =\sum_{i, j, k, l}\left(\sum_{m} h_{i m} R_{m j k l}+\sum_{m} h_{m j} R_{m i k l}\right)^{2} \\
& =\sum_{i, j, k, l}\left(\lambda_{i}-\lambda_{j}\right)^{2} R_{i j k l}^{2} \\
& =2 \sum_{i, j}\left(\lambda_{i}-\lambda_{j}\right)^{2}\left(1+\lambda_{i} \lambda_{j}\right)^{2} \\
& =4\left(S f_{4}-f_{3}^{2}-S^{2}-S(S-n)-n^{2} H^{2}+2 n H f_{3}\right),
\end{aligned}
$$

we have

$$
\begin{aligned}
\sum_{i, j, k, l} h_{i j k l}^{2} & =\frac{1}{4} \sum_{i, j, k, l}\left(h_{i j k l}-h_{i j l k}\right)^{2}+\frac{1}{4} \sum_{i, j, k, l}\left(h_{i j k l}+h_{i j l k}\right)^{2} \\
& =\frac{1}{4} \sum_{i, j, k, l}\left(h_{i j k l}-h_{i j l k}\right)^{2}+\frac{1}{16} \sum_{i, j, k, l}\left(h_{i j k l}+h_{i j l k}-h_{k l i j}-h_{k l j i}\right)^{2}+\sum_{i, j, k, l} u_{i j k l}^{2} \\
& =\frac{1}{4} \sum_{i, j, k, l}\left(h_{i j k l}-h_{i j l k}\right)^{2}+\frac{1}{16} \sum_{i, j, k, l}\left(h_{i j k l}-h_{k l i j}\right)^{2}+\frac{1}{16} \sum_{i, j, k, l}\left(h_{i j l k}-h_{k l j i}\right)^{2}+\sum_{i, j, k, l} u_{i j k l}^{2} \\
& =\frac{3}{8} \sum_{i, j, k, l}\left(h_{i j k l}-h_{i j l k}\right)^{2}+\sum_{i, j, k, l} u_{i j k l}^{2} \\
& =\sum_{i, j, k, l} u_{i j k l}^{2}+\frac{3}{2}\left(S f_{4}-f_{3}^{2}-S^{2}-S(S-n)-n^{2} H^{2}+2 n H f_{3}\right),
\end{aligned}
$$

where $u_{i j k l}=\frac{1}{4}\left(h_{i j k l}+h_{j k l i}+h_{k l i j}+h_{l i j k}\right)$.

From Ricci identities, we obtain that

$$
\sum_{j=1}^{n} u_{i i j j}=\frac{1}{2}\left[(n-S) \lambda_{i}+n H \lambda_{i}^{2}-n H\right],
$$

then by setting $a_{i j}=u_{i i j j}$, we have

$$
\begin{aligned}
\sum_{i, j, k, l} u_{i j k l}^{2} & \geq \sum_{i} a_{i i}^{2}+3 \sum_{i, j} a_{i j}^{2} \\
& \geq \frac{3}{2(n+4)}\left[(n-S)^{2} S+n^{2} H^{2} f_{4}-n^{3} H^{2}+2(n-S) n H f_{3}\right] .
\end{aligned}
$$

Combining (3.3) and (3.5), we obtain (3.1). 
LEMMA 3.3. Let $M^{n}$ be an $n$-dimensional $(n \leq 8)$ closed hypersurface with constant mean curvature $H$ in $\mathbb{S}^{n+1}(1)$. Then there exists a positive constant $\delta(n)<\min \left\{\frac{1}{2}, \frac{3}{n}\right\}$ depending only on $n$ such that

$$
3(A-2 B) \leq(2+\delta(n))\left(S-n H^{2}\right) \sum_{i, j, k} h_{i j k}^{2}+3 H^{2} \sum_{i, j, k} h_{i j k}^{2}-6 H \sum_{i, j, k} h_{i j k}^{2} \lambda_{i} .
$$

Proof. Let

$$
\mu_{i}=\lambda_{i}-H, \quad 1 \leq i \leq n
$$

then

$$
\begin{aligned}
3(A-2 B) & =3\left(\sum_{i, j, k} h_{i j k}^{2} \lambda_{i}^{2}-2 \sum_{i, j, k} h_{i j k}^{2} \lambda_{i} \lambda_{j}\right) \\
& =3\left(\sum_{i, j, k} h_{i j k}^{2}\left(\mu_{i}+H\right)^{2}-2 \sum_{i, j, k} h_{i j k}^{2}\left(\mu_{i}+H\right)\left(\mu_{j}+H\right)\right) \\
& =3\left(\sum_{i, j, k} h_{i j k}^{2} \mu_{i}^{2}-2 \sum_{i, j, k} h_{i j k}^{2} \mu_{i} \mu_{j}\right)+3 H^{2} \sum_{i, j, k} h_{i j k}^{2}-6 H \sum_{i, j, k} h_{i j k}^{2} \lambda_{i} \\
& \leq(2+\delta(n)) \sum_{i} \mu_{i}^{2} \sum_{i, j, k} h_{i j k}^{2}+3 H^{2} \sum_{i, j, k} h_{i j k}^{2}-6 H \sum_{i, j, k} h_{i j k}^{2} \lambda_{i} \\
& =(2+\delta(n))\left(S-n H^{2}\right) \sum_{i, j, k} h_{i j k}^{2}+3 H^{2} \sum_{i, j, k} h_{i j k}^{2}-6 H \sum_{i, j, k} h_{i j k}^{2} \lambda_{i},
\end{aligned}
$$

where the above inequality follows from Lemma 3.4 in [20], as $\sum_{i} \mu_{i}=0$.

THEOREM 3.4. Let $M^{n}$ be an $n$-dimensional $(n \leq 8)$ closed hypersurface with constant mean curvature $H$ in $\mathbb{S}^{n+1}(1)$ and $S$ be the length of the second fundamental form of $M^{n}$. Then there exist positive constants $\varepsilon(n)$ depending only on $n$, and $\delta(n, H)$ depending only on $n$ and $H$ such that if

$$
|H| \leq \varepsilon(n) \quad \text { and } \quad S_{0} \leq S \leq S_{0}+\delta(n, H),
$$

where

$$
S_{0}=n+\frac{n^{3}}{2(n-1)} H^{2}+\frac{n(n-2)}{2(n-1)} \sqrt{n^{2} H^{4}+4(n-1) H^{2}},
$$

then $S \equiv S_{0}$ and $M^{n}$ is isometric to the Clifford hypersurface. To be precise, $M^{n}$ is isometric to the Clifford torus $\mathbb{S}^{k}\left(\sqrt{\frac{k}{n}}\right) \times \mathbb{S}^{n-k}\left(\sqrt{\frac{n-k}{n}}\right)$ if $H=0 ; M^{n}$ is isometric to the Clifford hypersurface $\mathbb{S}^{1}\left(\frac{1}{\sqrt{1+\lambda^{2}}}\right) \times \mathbb{S}^{n-1}\left(\frac{\lambda}{\sqrt{1+\lambda^{2}}}\right)$ if $H \neq 0$.

Proof. By using (2.1), (2.2) and (2.3) and direct computations, we have

$$
\int_{M} \sum_{i, j, k} h_{i j k}^{2}=\int_{M}-S(n-S)+n^{2} H^{2}-n H f_{3},
$$




$$
\begin{gathered}
-\frac{1}{2} \int_{M}|\nabla S|^{2}=\int_{M} S^{2}(n-S)-n^{2} H^{2} S+n H f_{3} S+S \sum_{i, j, k} h_{i j k}^{2}, \\
\int_{M}-3 \sum_{i, j, k} h_{i j k}^{2} \lambda_{i}=\frac{3}{2} \int_{M}(n-S) f_{3}+n H f_{4}-n H S, \\
\int_{M} \sum_{i, j, k, l} h_{i j k l}^{2}=\int_{M}(S-2 n-3) \sum_{i, j, k} h_{i j k}^{2}+3(A-2 B)+\frac{3}{2}|\nabla S|^{2}-3 n H \sum_{i, j, k} h_{i j k}^{2} \lambda_{i} .
\end{gathered}
$$

Applying (3.1) to (3.12), we can obtain

$$
\begin{aligned}
& \int_{M}(S-2 n-3) \sum_{i, j, k} h_{i \ddot{k} k}^{2}+3(A-2 B)+\frac{3}{2}|\nabla S|^{2}-3 n H \sum_{i, j, k} h_{i j k}^{2} \lambda_{i} \\
& \geq \int_{M} \frac{3}{2}\left(S f_{4}-f_{3}^{2}-S^{2}-S(S-n)-n^{2} H^{2}+2 n H f_{3}\right) \\
& \quad+\int_{M} \frac{3}{2(n+4)}\left[(n-S)^{2} S+n^{2} H^{2} f_{4}-n^{3} H^{2}+2(n-S) n H f_{3}\right] .
\end{aligned}
$$

Combining (2.4), (3.9), (3.10) and (3.11) we get

$$
\begin{gathered}
\int_{M}-\left(\frac{5}{4} S-\frac{n}{4}+\frac{3}{2}\right)|\nabla h|^{2}+\frac{3}{2}(A-2 B)-\left(3-\frac{3}{n+4}\right) n H \sum_{i, j, k} h_{i j k}^{2} \lambda_{i} \\
+\int_{M} \frac{9 n+30}{4(n+4)}\left[-S^{2}(n-S)+n^{2} H^{2} S-n|\nabla h|^{2}-n H S f_{3}\right] \geq 0 .
\end{gathered}
$$

By (3.6), we have

$$
\begin{aligned}
& \int_{M}-\left(\frac{5}{4} S-\frac{n}{4}+\frac{3}{2}\right)|\nabla h|^{2}-\left(3 n+3-\frac{3 n}{n+4}\right) H \sum_{i, j, k} h_{i j k}^{2} \lambda_{i} \\
& +\int_{M} \frac{9 n+30}{4(n+4)} S\left[-S(n-S)+n^{2} H^{2}-n H f_{3}\right]-\frac{9 n+30}{4(n+4)} n|\nabla h|^{2} \\
& +\int_{M} \frac{2+\delta}{2}\left(S-n H^{2}\right)|\nabla h|^{2}+\frac{3}{2} H^{2}|\nabla h|^{2} \geq 0 .
\end{aligned}
$$

Since $S \geq S_{0}$, then by direct computation or [6], it is not difficult to get $-S(n-$ $S)+n^{2} H^{2}-n H f_{3} \geq 0$, and, moreover, if $S_{0} \leq S \leq S_{0}+\delta(n, H)$, the inequality (3.14) implies

$$
\begin{aligned}
& \int_{M}-\left(\frac{5}{4} S-\frac{n}{4}+\frac{3}{2}\right)|\nabla h|^{2}-\left(3 n+3-\frac{3 n}{n+4}\right) H \sum_{i, j, k} h_{i j k}^{2} \lambda_{i} \\
& +\frac{9 n+30}{4(n+4)}\left(S_{0}+\delta(n, H)-n\right)|\nabla h|^{2}+\frac{2+\delta}{2}\left(S-n H^{2}\right)|\nabla h|^{2}+\frac{3}{2} H^{2}|\nabla h|^{2} \geq 0 .
\end{aligned}
$$


Because $\sum_{i, j, k} h_{i j k}^{2} \lambda_{i} \leq \sqrt{S}|\nabla h|^{2}$, this means

$$
\begin{aligned}
& \int_{M}-\left(\frac{5}{4} S-\frac{n}{4}+\frac{3}{2}\right)|\nabla h|^{2}+\left(3 n+3-\frac{3 n}{n+4}\right) H \sqrt{S}|\nabla h|^{2} \\
& +\frac{9 n+30}{4(n+4)}\left(S_{0}+\delta(n, H)-n\right)|\nabla h|^{2}+\frac{2+\delta}{2}\left(S-n H^{2}\right)|\nabla h|^{2}+\frac{3}{2} H^{2}|\nabla h|^{2} \geq 0 .
\end{aligned}
$$

By the definition of $S_{0}$, and noting that $\delta<\frac{3}{n}$ and $|H| \leq \varepsilon(n)$ if $\varepsilon(n)$ is small enough, we can choose $\delta(n, H)>0$ such that

$$
\begin{aligned}
- & \left(\frac{5}{4} S-\frac{n}{4}+\frac{3}{2}\right)+\left(3 n+3-\frac{3 n}{n+4}\right) H \sqrt{S} \\
& +\frac{9 n+30}{4(n+4)}\left(S_{0}+\delta(n, H)-n\right)+\frac{2+\delta}{2}\left(S-n H^{2}\right)+\frac{3}{2} H^{2}<0 .
\end{aligned}
$$

According to (3.16) and the above inequality, we conclude that $|\nabla h| \equiv 0$. Hence, all of the above inequalities are equalities. Therefore, $-S(n-S)+n^{2} H^{2}-n H f_{3}=0$, which implies $S=S_{0}$ and $M$ is isometric to the Clifford hypersurface. Thus, the proof is completed.

AcKnowledgement. The authors would like to thank the referee for the careful reading of the manuscript, the valuable comments and useful suggestions.

\section{REFERENCES}

1. S. Almeida and F. Brito, Closed 3-dimensional hypersurfaces with constant with constant mean curvature and constant scalar curvature, Duke Math. J. 61 (1990), 195-206.

2. S. Almeida, F. Brito and L. A. M. Sousa, Jr., Closed hypersurfaces of $S^{4}$ with two constant curvature functions, Results Math. 50 (2007), 17-26.

3. E. Cartan, Sur des familles remarquables d'hypersurfaces isoparamétriques dans les espaces sphériques, Math. Z. 45 (1939), 335-367.

4. S. P. Chang, A closed hypersurface with constant scalar curvature and constant mean curvature in $\mathbb{S}^{4}$ is isoparametric, Comm. Anal. Geom. 1 (1993), 71-100.

5. S. P. Chang, On minimal hypersurfaces with constant scalar curvatures in $S^{4}, J$. Diff. Geom. 37 (1993), 523-534.

6. Q. M. Cheng, Y. J. He and H. Z. Li, Scalar curvature of hypersurfaces with constant mean curvature in a sphere, Glasg. Math. J. 51(2) (2009), 413-423.

7. S. S. Chern, M. Do Carmo and S. Kobayashi, Minimal submanifolds of a sphere with second fundamental form of constant length, in Functional analysis and related fields (Browder F. E., Editor) (Springer-Verlag Berlin, 1970), pp. 59-75.

8. W. Y. Hsiang, Remarks on closed minimal submanifolds in the standard riemannian m-Sphere, J. Diff. Geom. 1 (1967), 257-267.

9. H. B. Lawson, Jr., Local rigidity theorems for minimal hypersurfaces, Ann. Math. 89 (1969), 167-179.

10. T. Lusala, M. Scherfner and L. A. M. Sousa Jr., Closed minimal Willmore hypersurfaces of $S^{5}(1)$ with constant scalar curvature, Asian J. Math. 9(1) (2005), 65-78. 
11. C. K. Peng and C. L. Terng, Minimal hypersurfaces of spheres with constant scalar curvature. Seminar on minimal submanifolds, Ann. Math. Stud. 103 (1983), 177-198 (Princeton University Press, Princeton, NJ).

12. C. K. Peng and C. L. Terng, The scalar curvature of minimal hypersurfaces in spheres, Math. Ann. 266(1) (1983), 105-113.

13. M. Scherfner and S. Weiss, Towards a proof of the Chern conjecture for isoparametric hypersurfaces in spheres, Proceedings of 33rd South German Colloquium on Differential Geometry, TU, Vienna (2008), 1-33.

14. J. Simons, Minimal varieties in Riemannian manifolds, Ann. Math. 88 (1968), 62-105.

15. Y. J. Suh and H. Y. Yang, The scalar curvature of minimal hypersurfaces in a unit sphere, Commun. Contemp. Math. 9(2) (2007), 183-200.

16. S. M. Wei and H. W. Xu, Scalar curvature of minimal hypersurfaces in a sphere, Math. Res. Lett. 14(3) (2007), 423-432.

17. H. C. Yang and Q. M. Cheng, A note on the pinching constant of minimal hypersurfaces with constant scalar curvature in the unit sphere, Chinese Sci. Bull. 36 (1991), 1-6.

18. H. C. Yang and Q. M. Cheng, An estimate of the pinching constant of minimal hypersurfaces with constant scalar curvature in the unit sphere, Manuscripta Math. 82 (1994), 89-100.

19. H. C. Yang and Q. M. Cheng, Chern's conjecture on minimal hypersurfaces, Math. Z. 227 (1998), 377-390.

20. Q. Zhang, The pinching constant of minimal hypersurfaces in the unit spheres, Proc. Amer. Math. Soc. 138(5) (2010), 1833-1841. 